\title{
Tangence
}

\section{Souvenirs de lecture et souvenirs de soi : autobiographie et roman en face-à-face dans Le jardin des Plantes (1997) et Le tramway (2001) de Claude Simon Memories of reading and memories of self: autobiography meets fiction in Claude Simon's Le jardin des plantes (1997) and Le tramway (2001)}

\section{Katerine Gosselin}

Numéro 120, 2019

Le devenir-souvenir du roman. Poétique de la lecture romanesque

URI : https://id.erudit.org/iderudit/1069141ar

DOI : https://doi.org/10.7202/1069141ar

Aller au sommaire du numéro

Éditeur(s)

Tangence

ISSN

1189-4563 (imprimé)

1710-0305 (numérique)

Découvrir la revue

Citer cet article

Gosselin, K. (2019). Souvenirs de lecture et souvenirs de soi : autobiographie et roman en face-à-face dans Le jardin des Plantes (1997) et Le tramway (2001) de Claude Simon. Tangence, (120), 37-64. https://doi.org/10.7202/1069141ar

\section{Résumé de l'article}

Cet article étudie comme un diptyque les deux derniers romans de Claude Simon, Le jardin des Plantes (1997) et Le tramway (2001). Il y est montré comment le souvenir de roman et le souvenir personnel sont liés par une même dimension iconique ou un même devenir-image, en vertu duquel ils peuvent se substituer l'un à l'autre dans la mémoire ou se contaminer réciproquement. Cette contamination est appréhendée comme une perte ou une menace pour l'intégrité des souvenirs et de l'individu aussi bien que du texte romanesque : si un souvenir de roman peut tenir lieu et place du vécu dans la mémoire et déposséder en quelque sorte un individu de son passé propre, un souvenir personnel peut tout aussi bien prendre la place du roman dans la conscience et empêcher sa lecture. L'article veut montrer comment la confusion entre souvenirs de romans et souvenirs de soi est saisie dans Le jardin des Plantes comme une chance, le texte romanesque pouvant dès lors servir de copie de secours à la mémoire autobiographique, qu'il a d'ores et déjà infiltrée. Ainsi sont interprétées les longues citations de Sodome et Gomorrhe de Proust insérées dans Le jardin des Plantes, mais aussi l'abandon de la citation proustienne dans Le tramway au profit de l'image, marquant les limites ou une forme de deuil du roman. 
Tangence $\longrightarrow \mathrm{n}^{\circ}$ 120, 2019 p. 37-64.

\section{Souvenirs de lecture et souvenirs de soi: autobiographie et roman en face-à-face dans Le jardin des Plantes (1997) et Le tramway (2001) de Claude Simon \\ Katerine Gosselin \\ Université du Québec à Rimouski}

Si l'on présente communément Claude Simon comme un écrivain de la mémoire, il faut rappeler le travail de la critique qui, depuis les années 1990, s'est efforcée de montrer combien la mémoire est chez lui problématique, en crise; mémoire inquiète, comme l'affirmait Dominique Viart en 1997 dans un essai inaugural ${ }^{1}$, mais plus encore mémoire en défaut ou mémoire perdue, comme le concluait Isabelle Daunais en 2004:

À première vue, l'œuvre de Claude Simon a tout pour s'inscrire dans l'espace des voix mémorielles fortes, avec ses personnages obsédés de souvenirs, cherchant à rescaper de l'oubli et du néant des détails toujours plus fins et plus ténus. Mais cette mémoire est bien chez Simon [...] un processus ou une activité mentale plutôt qu'un espace où se seraient déposées les couches du monde, un savoir accumulé. La mémoire des personnages de Simon, ne se distingue pas d'une perception au présent et ne permet donc pas d'opérer la distance entre la réalité telle qu'elle est et une cohérence antérieure [...]. De sorte que la mémoire est ce qui manque au héros, ce qui lui échappe et qu'il s'efforce de rattraper [...]. La mémoire chez Simon est une mémoire en défaut ou une mémoire perdue ${ }^{2}$.

1. Dominique Viart, Une mémoire inquiète. La route des Flandres de Claude Simon, Paris, Presses universitaires de France, coll. «Écrivains», 1997.

2. Isabelle Daunais, "Nous étions à l'étude": mémoire et voix narrative», dans Marie-Pascale Huglo et Sarah Rocheville (dir.), Raconter? Les enjeux de la voix 
Les personnages de Simon, en effet, sont bien plutôt dépossédés de leur mémoire, dont ils tentent en vain de reconstituer quelques pans, se heurtant ultimement à «l'impalpable [...] brouillard de la mémoire ${ }^{3}$ ", selon les derniers mots du Tramway (2001).

Que la mémoire chez Simon «ne se distingue pas d'une perception au présent», comme l'écrit Isabelle Daunais, c'est bien ce qu'affirme Simon lui-même dans un passage fréquemment cité du Discours de Stockholm: «[L]'on n'écrit (ou ne décrit) jamais quelque chose qui s'est passé avant le travail d'écrire, mais bien ce qui se produit $[. .$.$] au cours de ce travail, au présent de celui-ci { }^{4} »$. Simon en donne pour exemple Stendhal prévenant le lecteur, dans Vie de Henry Brulard, d'un "danger de mensonge ${ }^{5}$ » dans ce qui est pourtant son «véridique journal ${ }^{6}{ }$. Alors qu'il raconte sa traversée du col du Grand Saint-Bernard avec l'armée d'Italie en 1800, Stendhal doute de l'authenticité de son souvenir, craignant que celui d'une gravure «fort ressemblante» contemplée cinq ou six ans après les événements s'y soit substitué, ait «pris [en lui] la place de la réalité»: «[E]t mon souvenir, conclut Stendhal, n'est plus que la gravure ${ }^{7}$.» Plus éloigné encore de l'événement originel, c'est, commente Simon dans le Discours de Stockholm, non pas même cette gravure que Stendhal devait décrire en écrivant, mais l'«image qui se formait alors en lui et qui prenait encore la place de la gravure ${ }^{8} »$, laquelle prenait déjà la place du souvenir. Nous sommes bien dans les méandres d'une mémoire perdue, ou irrémédiablement éloignée de son origine.

L'interrogation de Stendhal sur le référent autobiographique ou l'expérience vécue, laquelle serait invariablement et indéfiniment altérée dans le processus de remémoration, expose par la bande la

narrative dans le récit contemporain, Paris, L'Harmattan, 2004, p. 36; l'auteure souligne.

3. Claude Simon, Le tramway [2001], Paris, Minuit, coll. «Double», 2007, p. 141. Désormais, les références à cet ouvrage seront indiquées par le sigle $T$, suivi de la page, et placées entre parenthèses dans le corps du texte.

4. Claude Simon, Discours de Stockholm, dans Euvres, éd. Alastair B. Duncan, Paris, Gallimard, coll. «Bibliothèque de la Pléiade», 2006, t. I, p. 898; l'auteur souligne.

5. Stendhal, Vie de Henry Brulard, dans Euvres intimes, éd. Victor Del Litto, Paris, Gallimard, coll. «Bibliothèque de la Pléiade», 1982, t. II, p. 941.

6. Stendhal, Vie de Henry Brulard, ouvr. cité, p. 941.

7. Stendhal, Vie de Henry Brulard, ouvr. cité, p. 941; les citations comprises dans cette phrase sont toutes extraites de cet ouvrage en cette même page; les italiques sont de Stendhal.

8. Claude Simon, Discours de Stockholm, ouvr. cité, p. 899. 
dimension iconique des souvenirs: d'une traversée longue et périlleuse, qui fut très certainement marquante, Stendhal ne retient qu'une image figée. Si la mémoire chez Claude Simon est «en défaut» ou "perdue», c'est d'abord en raison de cette cristallisation du vécu en images figées, qu'aucun récit ne parvient plus à réintégrer dans une «cohérence antérieure», laquelle serait alors susceptible de constituer ou de fonder une mémoire.

Ce contraste entre la temporalité de l'expérience et l'iconicité des souvenirs hante les deux derniers romans de Simon, Le jardin des Plantes $^{9}$ (1997) et Le tramway (2001), où il caractérise indistinctement les souvenirs autobiographiques et les souvenirs de romans, tous deux réduisant un temps traversé, temps de la lecture ou temps de la vie, à une poignée d'images. Dans le cadre de ce dossier sur la poétique de la lecture romanesque, le cas de Simon permettra ainsi de définir le devenir-souvenir du roman comme un devenir-image, en vertu duquel il se confond avec le vécu dans la mémoire, l'un se substituant à l'autre et inversement. Troublante pour le brouillage qu'elle opère entre fiction et réalité, menaçante pour l'individu qui tente de se saisir de son identité et de son passé par l'écriture autobiographique, nous verrons comment cette confusion devient dans Le jardin des Plantes et Le tramway une chance, offrant la possibilité de remonter, à même le texte romanesque, le cours de l'expérience vécue, ou de la mémoire perdue.

\section{Quand le souvenir de roman se substitue au vécu}

Le jardin des Plantes est un roman extrêmement éclaté, constitué de la juxtaposition de fragments les plus hétérogènes (récits, citations, ekphrasis, extraits d'archives, de manuscrits, de mémoires, etc.), au fil desquels se dégage cependant une trame principale, d'où pourrait émaner, par association mémorielle ou métaphorique, l'ensemble des fragments rassemblés. Cette trame est fort simple, et se déroule en une heure à peine: elle rassemble un écrivain nobélisé, désigné comme «S.», et un journaliste venu l'interviewer dans son appartement du $5^{\mathrm{e}}$ arrondissement à Paris. L'objet de l'entretien est l'expérience de la débâcle française de mai 1940, à laquelle l'écrivain S., alors brigadier dans un régiment de dragons, a miraculeusement

9. Claude Simon, Le jardin des Plantes, Paris, Minuit, 1997. Désormais, les références à cet ouvrage seront indiquées par le signe $J P$, suivi de la page, et placées entre parenthèses dans le corps du texte. 
survécu, dans une suite d'événements qu'il a racontée dans ses différents romans ${ }^{10}$. L'objectif du journaliste est de soutirer le témoignage de S., de l'arracher à la «sauce romanesque» (JP, p. 272) dans laquelle le romancier aurait selon lui enrobé «les faits bruts» ( $J P$, p. 272). À défaut d'atteindre cet objectif — la sauce romanesque tache - , le journaliste, dans une sorte de maïeutique ${ }^{11}$, amènera $S$. à produire un nouveau récit des événements de mai 1940, où un élément fondamental, inattendu de son expérience ressurgira.

Cet élément inattendu, c'est la mélancolie que $\mathrm{S}$. avoue avoir ressentie devant l'imminence de la mort, dans une sorte d'anachronisme que signalent les clichés romantiques qu'il en vient à interpeler:

[E] tà la fin il dit Mélancolie,le journaliste s'exclamant Mélancolie!... [...] Le journaliste répétant: Mélancolie! Simplement mélancolie? Vous... et S. disant Oui. Simplement, [...] disant que probablement pour le journaliste, comme d'une façon générale, le mot mélancolie doit faire surgir [des] images plus ou moins mièvres [...] [mais] S. dit que c'était $[\ldots]$ exactement le contraire: [...] quelque chose de violent qui protestait, furieux, bâillonné mais hurlant: Jamais je n'avais tant désiré vivre, jamais je n'avais regardé avec autant d'avidité, d'émerveillement, le ciel, les nuages, les prés, les haies... (JP, p. 299-303)

10. Dans un article sur Le jardin des Plantes, Michèle Touret rappelle que cette interview est un autre "document» inséré dans le roman, renvoyant pour le lecteur à «l'interview réelle que Claude Simon a donnée au journaliste du Figaro Pierre Bois, et qui avait paru le 13 juillet 1990, première d'une série consacrée à la défaite de 1940 et à ses suites» ("L'autre moitié de l'orange, la guerre cinquante ans après», dans Paul Dirkx et Pascal Mougin (dir.), Claude Simon: situations, Lyon, ENS Éditions, coll. «Signe», 2011, p. 152). Publié sous le titre «La déroute des Flandres», l'entretien a été réédité dans le deuxième tome des œuvres complètes de Simon dans la collection de la Pléiade (Claude Simon, «La déroute des Flandres», dans Euvres, éd. Alastair B. Duncan, Paris, Gallimard, coll. «Bibliothèque de la Pléiade», 2011, t. II, p. 1368-1378).

11. Dans un article paru peu près la publication du Jardin des Plantes, Didier Alexandre ( «L'enregistrement du Jardin des Plantes», Littératures, n 40 (Claude Simon: Le jardin des Plantes), printemps 1999, p. 10) présente le journaliste comme "celui qui accouche $\mathrm{S}$. de sa vérité». L'article a été réédité sous le même titre dans le récent numéro des Cahiers Claude Simon consacré au Jardin des Plantes (Cahiers Claude Simon, $\mathrm{n}^{\circ} 13$ (Le jardin des Plantes. Fragments, lopins, parcelles), 2018, p. 95-109). Les citations ultérieures à cet article renverront à la publication originale dans Littératures. 
Dans un numéro précédent de Tangence, j'ai eu l'occasion de retracer les étapes de la remontée vers cette mélancolie romantique dans Le jardin des Plantes, rendue possible par le déploiement d'un vaste réseau intertextuel qui, par le biais de La route des Flandres (1960) et de La corde raide (1947) de Simon, mène jusqu'à La chartreuse de Parme (1839) de Stendhal ${ }^{12}$.

L'intertexte stendhalien n'est pas le plus visible dans Le jardin des Plantes (des écrivains comme Proust ou Dostoïevski sont cités et commentés avec beaucoup plus d'insistance), mais il n'en est pas moins explicite. Stendhal, en effet, est le premier romancier cité, si l'on fait exception de l'exergue, et son nom apparaît de surcroît dans le fragment où est introduit l'entretien avec le journaliste qui donne sa trame principale au roman, où il intervient cependant comme un repoussoir:

Au galop penché sur l'encolure je pensais naïvement les maladroits ils tirent trop bas [...] De temps à autre un cavalier tombait de son cheval ou roulait par terre avec lui mais on ne se retournait pas Des années plus tard un journaliste m'a demandé comment on faisait pour vivre avec la peur Je lui ai dit Vous voyez chacun fait ce qu'il peut ce n'est pas comme Stendhal caracolant gaiement sous les boulets à Waterloo. Il m'a dit Pas Stendhal: Fabrice. J'ai dit Mais si Mais si Fabrice suivait lui dans les fourgons de l'intendance vous pensez bien que Stendhal n'a pas manqué cette occasion de montrer ce courage dont il était si soucieux (JP, p. 34-35).

Plus tard dans l'entretien, lorsqu'il tentera d'expliquer au journaliste comment il s'est senti après l'effondrement de son régiment, en avançant à découvert sur la route derrière son colonel qui les menait, lui et deux autres survivants, à une mort certaine, S. utilisera une autre référence stendhalienne:

S. dit au journaliste que lorsque l'on conduit Julien Sorel à la guillotine Stendhal écrit que celui-ci se trouve "en veine de courage» et que «marcher au grand air est pour lui une sensation délicieuse» (sapristi comment peut-on écrire — et qui plus est lire de pareilles fadaises!), tandis que le «grand air» où se déroulait la marche des quatre cavaliers (l'éclatant soleil, la paisible campagne,

12. Voir Katerine Gosselin, «À contretemps du Nouveau roman. Réécriture et relecture dans Le jardin des Plantes de Claude Simon», Tangence, $\mathrm{n}^{\circ} 107$ (Des communautés de lecteurs, dir. Jean-François Hamel et Julien Lefort-Favreau), 2015, p. 33-54. 
les pépiements d'oiseaux) rendait au contraire la chose disons... infiniment plus disons... insupportable qu'elle ne l'aurait été si tout cela s'était passé de nuit, ou sous la pluie (JP, p. 297-298).

Comme j'ai tenté de le montrer dans l'article précédemment cité, le déni de Julien Sorel masque ici, en même temps qu'il la désigne, une allégeance fondamentale à un autre personnage stendhalien, celui que le journaliste avait initialement nommé: Fabrice del Dongo. $\mathrm{Si}$, en 1997, cette allégeance est inavouable pour l'écrivain devenu, quelque dix ans après la guerre, un Nouveau romancier, pourfendeur du réalisme et du naturalisme aux côtés des Alain Robbe-Grillet et Jean Ricardou ${ }^{13}$, elle était pourtant avouée très clairement dans le premier livre écrit par Simon après la guerre, paru en 1947, La corde raide ${ }^{14}$, qu'il a par ailleurs renié par la suite ${ }^{15}$.

La corde raide est un texte inclassable, entre récit autobiographique et essai, où Simon livre des souvenirs de jeunesse, de la guerre d'Espagne et de la débâcle de 1940, mêlés aux réflexions d'ordre existentiel qu'ils suscitent dans le cours de la remémoration. Simon y situe explicitement, au cœur de son expérience de la débâcle, le souvenir d'un roman, plus précisément d'un personnage de roman, le même Fabrice del Dongo:

[J'étais alors] entré jusqu'à mi jambes dans l'eau d'un ruisseau, essayant désespérément de le faire franchir par ma jument, tandis qu'autour de moi craquaient de multiples petits nuages gris noir et que sur la route proche des tanks étaient en train de se démolir à coups de canon. J'eus le temps de me voir, de penser que je devais être ridicule, de me comparer à une gravure de chasse anglaise où un monsieur en habit rouge tirait de la même façon sur un cheval, de me souvenir de Fabrice del Dongo, et de penser que c'était de

13. Les deux représentants ou chefs de file, comme on les a souvent appelés, du Nouveau roman sont désignés respectivement dans Le jardin des Plantes par les initiales A. R.-G. et J. R. (JP, p. 356-358), introduites dans une longue citation des débats tenus à Cerisy en 1971 lors du colloque «Nouveau roman: hier, aujourd'hui ", organisé par Jean Ricardou, dont les actes ont été publiés en 1972 (Jean Ricardou et Françoise Van Rossum-Guyon (dir.), Nouveau roman: hier, aujourd'hui, Paris, Union générale d'éditions, coll. «10/18», 1972, 2 t.).

14. Claude Simon, La corde raide, Paris, Sagittaire, 1947.

15. À tout le moins, La corde raide "fait partie des textes dont [Simon] estimait qu'ils ne devaient pas être réédités», peut-on lire dans la notice de l'ouvrage sur le site de l'Association des lecteurs de Claude Simon [En ligne], mis en ligne le 10 septembre 2014, consulté le 12 novembre 2019, URL: http://associationclaudesimon.org/claude-simon/oeuvres/article/la-corde-raide-1947. 
cette manière ridicule que j'allais crever, participant à une grande bataille dont on parlerait plus tard (mensongèrement), dans les livres d'histoire ${ }^{16}$.

Ce que S., sur les rives de la Meuse en 1940, et Fabrice à Waterloo en 1815 ont en commun, c'est de n'être pas présent aux événements de la guerre, qui se déroulent, semble-t-il, en dehors d'eux, dans «une sorte de brouillard d'irréalité» (JP, p. 262). J'en concluais en 2015, m'appuyant sur les analyses de Tiphaine Samoyault dans La montre cassée $^{17}$ : "Comme Fabrice, [...] qui "n'eut guère la conscience de ce qui se passait aujourd'hui[, qui] se sentait fort las", contemplant un "spectacle curieux" auquel il ne "comprenait rien du tout", S. n'a pas été présent aux événements de mai 1940 », cette absence consacrant l'impossibilité de s'en souvenir et donc d'en témoigner, ou de produire un témoignage autre que celui de cette impossibilité même.

Ce que révèle la double référence à Fabrice dans La corde raide et Le jardin des Plantes, sur laquelle je me fonderai pour tenter une première caractérisation du devenir-souvenir du roman, c'est que le souvenir du roman, ici, tient lieu de l'expérience vécue: au moment le plus fort de la débâcle, au commencement de la fin, de la déroute, un souvenir de roman s'est interposé entre S. et les événements, $\mathrm{S}$. qui, en conséquence, ne pourra jamais revenir en arrière, se souvenir de son propre passé, qu'il n'a pour ainsi dire pas vécu — il s'est souvenu d'une gravure de chasse et de Fabrice en lieu et place. En cherchant à revenir à l'expérience originelle de S., le journaliste effectue bel et bien une forme de remontée; mais celle-ci, loin d'aboutir à une antériorité positive, lance plutôt le témoignage dans un jeu de miroirs où se perd l'origine.

C'est que les termes logiquement opposés de la vie et du roman ou de la réalité et de la fiction sont confondus dans l'expérience de S.:

16. Claude Simon, La corde raide, ouvr. cité, p. 53; je souligne. Sans mentionner Stendhal ni Fabrice cependant, Simon fait un récit similaire des événements dans l'entretien accordé à Pierre Bois pour Le Figaro en 1990 ( «La déroute des Flandres», dans Euvres, ouvr. cité, t. II, p. 1372): «Alors, à ce moment, a commencé une scène parfaitement grotesque: ma jument intéressée par deux étalons qui se trouvaient dans le pré refusant absolument de traverser ce ruisseau, moi pied à terre, entré dans l'eau jusqu'à mi-mollet, tirant en jurant sur les rennes (vous voyez le tableau: comme une de ces humoristiques gravures de chasse anglaises...) [...]. Je me rappelle avoir pensé: "Voilà: tu vas mourir de cette façon bouffonne et on écrira "Tombé au champ d'honneur"! »

17. Tiphaine Samoyault, La montre cassée, Lagrasse, Verdier, coll. "Chaoïd», 2004; voir p. 116-117 particulièrement. 
le souvenir du roman et l'expérience vécue sont pour lui identiques, en ce qu'ils se cristallisent en une seule et même image. C'est effectivement une image qui relie, pour S., les événements de la débâcle au souvenir de Fabrice, si l'on en croit l'extrait précédemment cité de La corde raide: "J'eus le temps de me voir, de penser que je devais être ridicule, de me comparer à une gravure de chasse anglaise où un monsieur en habit rouge tirait de la même façon sur un cheval, de me souvenir de Fabrice del Dongo ${ }^{18}{ } \ldots$... Entre le «je» et le souvenir de Fabrice, il y a une gravure, image au trait fixe s'il en est, qui tient ensemble les histoires du jeune Simon et de Fabrice, qui en fait les deux faces d'une même pièce.

Si cette image est ce qui permet de faire le lien entre les deux histoires, elle est aussi réduction violente de la matière romanesque. Épousant le temps de la vie même ${ }^{19}$, le roman subit dans nos mémoires le même sort que notre vécu ou notre passé propre: il se réduit en images, en quelques clichés photographiques que seul le récit peut remettre en mouvement, dans une démarche prospective et sans fin. Cette iconicité de la mémoire romanesque, S., dans Le jardin des Plantes, en fait l'expérience à ses dépens. Il relate en effet au journaliste sa surprise devant les jaquettes d'un de ses livres (que tout désigne comme étant La route des Flandres) produites par des éditeurs étrangers:

S’il vous plaît, justement[, interrompt le journaliste]: quand vous vous êtes trouvé sur cette route que vous avez si bien décrite dans votre roman et... J'ai dit Merci vous êtes bien gentil mais il faut croire que je ne l'ai pas si bien décrite, parce que figurezvous qu'un des éditeurs étrangers a trouvé bon [...] de décorer la jaquette du livre avec un dessin représentant le cadavre d'un cheval en travers d'une route défoncée, boueuse, parsemée de flaques et bordée d'arbres déchiquetés. Il n'y manquait que les classiques ruines, les classiques pans de murs eux aussi déchiquetés. Mais ça

18. Claude Simon, La corde raide, ouvr. cité, p. 53 ; je souligne.

19. Sur la façon dont le temps romanesque calque ou reproduit celui de la vie même, voir l'article de Christophe Pradeau, qui revient entre autres sur le débat entre Albert Thibaudet et Paul Bourget sur la composition romanesque: «Contre la notion de muthos, conclut Pradeau, Thibaudet affirme que le roman, comme d'ailleurs l'épopée, ne peut être une composition précisément parce qu'il est "de la vie qui se crée elle-même à travers une succession d'épisodes", épisodes qui sont, comme il l'écrit ailleurs, "autant de coupes sur le flux même de la vie"." ( «e roman a le temps», Poétique, n 132, novembre 2002, p. 390) 
aussi d'autres éditeurs y ont pensé. D’une manière générale les couleurs des jaquettes tirent sur le rouge et le noir. Feu et ténèbres. (JP, p. 100-101)

Les images de la guerre véhiculées par les éditeurs sont si clichées, souligne ironiquement S., si facilement reconnaissables qu'elles dispensent de la lecture du roman: "Comme ça le lecteur n'a plus besoin de lire, et si par malheur il lit il va être terriblement déçu parce que là (je veux dire sur cette route) s'il y avait de la fumée c'était juste de loin en loin, et juste un filet, et non pas noire mais bleutée dans le soleil, [...] et le ciel tout bleu» (JP, p. 101). Non seulement les images clichées des jaquettes tiennent-elles lieu et place du texte romanesque, que dès lors il n'est plus besoin de lire, mais, plus encore, elles trahissent l'expérience vécue que voulait «décrire ${ }^{20}$ » ce texte. Avec l'humilité de l'artisan, S. s'accuse finalement, plutôt que les lecteurs, de ces clichés suscités par son roman: «Alors j'ai sans doute mal raconté tout ça et il faudrait reprendre» (JP, p. 101). Reprendre le texte, encore une fois, pour tenter de le faire correspondre davantage à l'expérience, à la réalité vécue, si possible.

Cette reprise du geste d'écrire, dans un souci infatigable de justesse, Proust en est la figure tutélaire dans Le jardin des Plantes, lui dont Simon cite à plusieurs reprises la correspondance avec les éditions Gallimard. Dans le processus de révision d'À l'ombre des jeunes filles en fleurs (1919), Proust fait montre d'une attention extrême au détail, rendue encore plus évidente, ou plus inutile, sinon scandaleuse, insensée, par le contexte de guerre rappelé par Proust luimême dans un échange avec Gaston Gallimard (JP, p. 139²1):

20. Le terme renvoie au titre de travail de La route des Flandres, qui était, comme Simon l'a confié dès un entretien de 1960 accordé à Claude Sarraute pour Le Monde, «Description fragmentaire d'un désastre» ( Avec La route des Flandres, Claude Simon affirme sa manière», entretien avec Claude Sarraute, Le Monde, 8 octobre 1960 , p. 9 ).

21. Dans l'extrait cité de la correspondance, Proust signale l'intérêt que son œuvre pourrait avoir pour les lecteurs en temps de guerre, contre toute attente $(J P$, p. 139: l'omission dans la citation de la correspondance est le fait de Simon, signalée par des parenthèses que je reproduis telles quelles) : «Au mois d'octobre 1916, Proust écrit à Gaston Gallimard: "Et puisque ce mot de guerre est venu 'sous ma plume', je crois (mais d'ailleurs c'est sans intérêt pratique, puisque nous ne le pouvons pas) que j'ai eu tort de vouloir attendre la fin de la guerre pour paraître (...). Mais (mes raisons) maintenant que j'y ai pensé (et encore une fois c'est toujours théorique) sont qu'en ce moment où (pas moi mais presque tout le monde) on s'est habitué à la guerre, on ne lit guère que le communiqué et encore, on aimerait quelque chose d'autre, on pourrait s'intéresser 
«Pour "À l'ombre des jeunes filles en fleurs", serait-ce encore temps d'ajouter 3 lignes à une page et un infinitif à l'autre? J'en serais très heureux", écrit Proust le 25 juillet 1918 à Berthe Lemarié des Éditions Gallimard, et le $1^{\text {er }}$ août: «À la page 81 à la ligne 12 il y a: dans un tout petit vide, une fenêtre doublée de soleil; j'y restais une etc. Or je trouve ce "doublée de soleil" affreux et ce "j'y restais" pas français.

Je modifie donc ainsi la ligne 12: dans un tout petit vide, que commençait déjà à faire rêver l'après-midi bleu de ses fenêtres; je restais seul etc.» $(J P$, p. 141)

Deux forces contraires sont ainsi en lutte dans Le jardin des Plantes: une force de travail qui remet inlassablement sur le métier son ouvrage, reprenant sans cesse l'écriture du roman, dans un infini, dérisoire souci du détail pour l'amener au plus près de la réalité, et une force d'oubli qui réduit ce travail, sinon à néant, tout juste à quelques clichés dans la mémoire des lecteurs. L'espace de l'écriture romanesque, d'un côté, et l'espace de la mémoire, de l'autre côté, en lutte l'un contre l'autre. Stendhal, peut-être, s'accommodait mieux de cette lutte, lui dont Simon cite aussi dans Le jardin des Plantes cet extrait des manuscrits de Lucien Leuwen:

«Au plus fort de cet examen de conscience [et] de la folie de Mademoiselle Raimonde, la porte de la loge s'ouvrit pour donner passage à etc.»

En note dans la marge du manuscrit Stendhal écrit:

«Le lecteur qui connait les lieux lira: assise sur les genoux de Leuwen qui avait la main je ne sais où et allait la br... Faire comprendre: avait sur ses genoux.» (JP, p. 43-44)

«Faire comprendre» ou, plus exactement, «faire voir»: le romancier écrit quelque chose et fait voir autre chose. Sa lettre n'est qu'un voile soigneusement érigé mais destiné à se consumer. Stendhal semble avoir tiré profit de cette dissociation, ou accepté de tabler sur ses possibilités. À l'image de Proust tel qu'il le dépeint dans Le jardin des Plantes $^{22}$, révisant son travail avec un soin déconcertant, S. se

à une longue œuvre. Après la guerre, la Paix, la victoire, seront des choses nouvelles, savoureuses, on y pensera, plutôt qu'à lire. Et alors la guerre elle-même déjà rétrospective, deviendra l'objet d'un intérêt d'imagination qu'elle n'excitait pas comme réalité quotidienne et d'un progrès insensible.»

22. Je parle ici de Proust écrivain, assis à sa table de travail, pour reprendre une représentation courante de l'écrivain chez Simon. Des fragments de la première 
montre plus obstinément attaché à la lettre du texte, par laquelle il lutte contre le «brouillard d'irréalité» $(J P$, p. 262) qui perdure dans la mémoire.

Entre ces deux espaces de l'écriture et de la mémoire, cependant, l'image semble irréductible et devoir être le devenir de toutes choses: le romancier lutte pour raconter une expérience ou une réalité vécue déjà figée en images dans sa mémoire, images qu’il tente de remettre en mouvement, de réanimer... mais dont il ne restera, par-delà la transmission narrative, que quelques clichés dans la mémoire du lecteur. Plus qu'une faiblesse ou une faillite du roman, cet universel devenir-image signale chez Simon l'indistinction dans la mémoire entre le roman et la vie. Ce sont là les deux faces de l'iconicité des souvenirs de romans telle que la saisit Simon dans Le jardin des Plantes: elle dénie, fait sombrer dans un oubli profond «des pans entiers ${ }^{23}$ » du texte, comme l'écrit Julien Gracq dans En lisant en écrivant, mais, ce faisant, elle permet aux souvenirs de romans de prendre place dans la mémoire au côté des souvenirs personnels, dans une indistinction troublante, vertigineuse - mais peut-être aussi «protect[rice]» $(T$, p. 132), pour reprendre un des derniers mots du Tramway, que nous étudierons ci-après. C'est bien ce dont prend conscience $\mathrm{S}$. dans son échange avec le journaliste, lorsque sa tentative de témoignage, de «compte rendu» des «faits bruts», nettoyés de la «sauce romanesque», aboutit sur un souvenir de

partie du Jardin des Plantes portent en effet sur certains détails de la biographie de Proust qui en présentent une autre image, ce qui nous ramène peut-être à la dissociation avec la lettre: «Vers la fin de sa vie, Marcel Proust se faisait apporter dans le bordel pour hommes où il avait ses habitudes des rats qu'il s'amusait à tuer en les transperçant lentement à travers les barreaux de leur cage à l'aide de ces longues aiguilles à chapeaux comme en portaient les femmes à son époque [...]. Proust semblait éprouver un grand plaisir à leurs cris en même temps qu'au spectacle de leurs soubresauts et de leurs agonies.» (JP, p. 106) Sur ces fragments du Jardin des Plantes qui commentent la vie de Marcel Proust, voir Marie Miguet-Ollagnier, "Le jardin des Plantes à l'ombre de Marcel Proust», Bulletin d'informations proustiennes, $\mathrm{n}^{\circ} 29,1998$, p. 129-140.

23. Julien Gracq écrit: «Il faudrait comparer entre eux les souvenirs que gardent à distance d'une même ouvre des lecteurs exercés et de bonne foi, leur faire raconter de mémoire à leur idée le livre — ou plutôt ce qu'il en reste, toute référence au texte omise - noter la récurrence plus ou moins régulière du naufrage de pans entiers qui ont sombré dans le souvenir, de points d'ignition au contraire qui continuent à l'irradier, et à la lumière desquels l'ouvrage se recompose tout autrement.» (En lisant en écrivant [1980], dans CEuvres complètes, éd. Bernhild Boie, Paris, Gallimard, coll. «Bibliothèque de la Pléiade», 1995, t. II, p. 646; l'auteur souligne) 
roman: celui-ci a pris en lui «la place de la réalité», pour reprendre les termes de Stendhal cités en introduction. Témoigner du passé devient dès lors, dans une adéquation parfaite, dire ses souvenirs de romans: dire les romans dont on s'est souvenu à défaut d'avoir été présent aux événements.

Mais le roman n'est pas pour autant hégémonique. L'indistinction entre le roman et la vie, rendue possible par l'iconicité des souvenirs, fonctionne dans les deux sens. Si le roman peut se substituer au vécu dans la mémoire, le vécu peut de même se substituer au roman et l'incorporer. C'est ce que relate le narrateur simonien dans Le tramway, quatre ans après Le jardin des Plantes.

\section{Quand le souvenir de soi se substitue au roman}

Le tramway est un roman tout différent du Jardin des Plantes, beaucoup plus court, moins foisonnant, centré sur quelques expériences de l'enfance et de la vieillesse: les vacances estivales et les années de collège dans le sud de la France, dans l'après-Grande Guerre, marquées par la maladie et la mort prématurée de la mère, et, à l'autre bout du siècle, un séjour à l'hôpital où le vieil homme se remémore cette période de l'enfance. Le roman est composé de fragments d'une à plusieurs pages portant sur ces époques ou termes éloignés de la vie, entre lesquels il circule en accéléré, à la manière du tramway qui donne son titre au roman, et qui reliait la ville à la plage dans l'enfance du narrateur.

Un des souvenirs d'enfance raconté dans Le tramway concerne une fillette aux «manières garçonnières» qui «avait pris d'autorité le commandement» ( $T$, p. 49) du petit groupe d'amis du narrateur. Celui-ci garde le souvenir très vif d'une saynète jouée avec fougue et énergie par la fillette, qui l'avait alors fortement impressionné, suscitant en lui «une ferveur et un émerveillement muets mais non moins passionnés» $(T, \mathrm{p} .49)$ :

[D]éguisée en garçon et coiffée d'une casquette, elle faisait irruption [sur la scène] en sauveur, sautant d'un bond à l'intérieur d'un taudis londonien par une fenêtre dont je me souviens qu'elle était ménagée sur la droite, comme je crois encore entendre le bruit sourd du plancher sous le choc de ses pieds et voir le léger nuage de poussière qui s'en éleva. ( $T$, p. 49) 
Gravé dans la mémoire d'enfant du narrateur, ce souvenir ressurgira par la suite lors de sa lecture d'À l'ombre des jeunes filles en fleurs, dans une scène précise que le souvenir occultera, en quelque sorte:

[B]ien des années plus tard le souvenir de [1]a bruyante et salvatrice entrée sur scène [de la fillette] jouait encore si vivement qu'il me semblait (me semble encore) entendre ce bruit du plancher à son atterrissage et que, par la suite, je plaquais cette image sur celle du saut à pieds joints d'Andrée, la jeune compagne d'Albertine, par-dessus le "pauv' vieux» assis sur la promenade de Balbec. (T, p. 49-50)

Ainsi, s'il arrive qu'un souvenir de roman prenne la place du vécu, de la réalité, il arrive aussi qu'un souvenir personnel prenne la place du roman.

Si, dans l'expérience de la débâcle telle que rappelée par Le jardin des Plantes, un souvenir de roman a tenu lieu de l'expérience elle-même, a arraché le personnage à son présent, pourrions-nous dire (en même temps qu'il lui a permis d'y survivre, peut-être; nous reviendrons sur la nature traumatique de l'événement), c'est, dans cet extrait du Tramway, le souvenir personnel qui se substitue au roman, qui entraîne le texte du côté du lecteur. Souvenir personnel et texte romanesque sont littéralement substituables: il se produit, pour reprendre les termes de Marie Miguet-Ollagnier, «une véritable osmose entre la réminiscence littéraire et le souvenir personnel $^{24} »$. Le terme « osmose» n'est pas trop fort, mais il traduit mal la confusion et surtout la perte, la privation qu'engendre le phénomène osmotique: de la même façon que le souvenir du roman de Stendhal prive l'écrivain S. d'un accès à son passé dans Le jardin des Plantes (S. n'aura jamais accès à son souvenir réel de l'événement), le souvenir personnel empêche le narrateur du Tramway de lire le texte proustien, d'y avoir accès. En effet, il a beau lire et relire, en lisant, ce n'est pas Andrée qu'il voit à Balbec, mais la fillette de son enfance dans l'ambiance méditerranéenne des vacances familiales: le texte lui est devenu impénétrable, inaccessible. Le roman est ainsi doublement menacé, non seulement de devenir souvenir, d'être réduit à une image, mais, plus encore, d'être confondu dans ce devenir-image avec la mémoire personnelle.

24. Marie Miguet-Ollagnier, "Claude Simon face à Proust: exercices d'admiration », L'Esprit Créateur, vol. 46, n 4, 2006, p. 106. 
L'adhésion intime au texte proustien dont témoigne cette expérience de lecture est contredite dans un fragment subséquent du Tramway; ce développement métatextuel détonne avec le reste du roman par son ton mais aussi sa teneur critique:

On s'explique mal pourquoi, torturé par la jalousie, le narrateur (qui, comme Proust le laisse entendre, pourrait s'appeler Marcel) confie pour les après-midi la garde d'Albertine à cette Andrée dont (après l'observation de Cottard sur les deux jeunes filles dansant ensemble au Petit Casino) il soupçonne qu'elle a entretenu avec sa maîtresse une amitié particulière ou plutôt gomorrhéenne: mot [...] forgé [...] d'une façon assez malheureuse, évoquant phonétiquement logorrhée ou encore gonocoque. À noter du reste que les problèmes de vraisemblance n'embarrassent pas Marcel Proust qui, au besoin, recourt sans vergogne aux procédés les plus éculés (comme les hasards, les coïncidences, le fortuit) ( $T$, p. 54-55).

Le narrateur constate ensuite ce qui lui apparaît comme l'invraisemblance généralisée du portrait de société dressé par Proust, qu’il juge tel en le comparant avec la société dans laquelle il a grandi:

De même, on reste pour le moins étonné par le dramatique tableau que fait le même narrateur, au début de «Sodome et Gomorrhe", de certaines pratiques dont il présente longuement les adeptes maudits avant d'avertir le lecteur que ces «maudits» se comptent par milliers dans toute société [...]. Et, comme il en était de l'antisémitisme, je ne me souviens pas, au cours de mon enfance et dans le milieu des plus collet monté où j'ai été élevé, d'avoir entendu la moindre condamnation de ces pratiques dont le représentant le plus en vue appartenait lui-même à ce que l'on appelait (ou s'appelait elle-même) la «société» ( $T$, p. 55-56).

Peu avant, le narrateur du Tramway s'était en effet étonné des bonnes relations entretenues par sa mère et ses tantes avec une famille juive, celle de madame Espinosa, à qui la mère avait loué deux chambres dans sa villa, «la question de la judéité n'ayant, [confie-t-il,] tout au moins en [s]a présence, jamais été évoquée, même par allusion [...] dans ce milieu pourtant ultra-catholique et réactionnaire, soit qu'on la jugeât de mauvais goût ou encore, comme il semble, qu'elle n'eût tout simplement pas cours dans cet extrême sud de la France» (T, p. 43-44). 
De la même façon que le déni de Stendhal à l'entrée du Jardin des Plantes recouvre la mémoire profonde, souterraine et matricielle que garde S. de La chartreuse de Parme, le déni ou la critique de Proust dans Le tramway est un voile posé dérisoirement ou ironiquement sur la contamination des souvenirs du narrateur par le texte proustien. Car l'affirmation selon laquelle la société de La recherche ne serait pas vraisemblable est incongrue, jusqu'à un certain point illégitime. D'abord, les souvenirs d'enfance du Tramway se déroulent explicitement dans les années 1920, après la Première Guerre ${ }^{25}$, c'està-dire de vingt à trente ans après l'intrigue de La recherche du temps perdu, dont la plus grande part se déroule dans les années 1890 et 1900. Ensuite, le milieu d'où vient le narrateur du Tramway n'est pas le même que celui où gravitent les personnages de La recherche: s'il est politiquement aussi réactionnaire, il est socio-économiquement moins aisé, moins élevé dans la hiérarchie sociale et marqué géographiquement par son appartenance à la province. Ces différences historiques, sociales, économiques et géographiques pourraient très bien, en toute rigueur, expliquer «l'écart» entre la société représentée par Proust et celle dans laquelle a grandi le narrateur du Tramway, et ainsi dédouaner Proust de ce qui semble ses invraisemblances.

En argumentant ainsi, nous jouons cependant le jeu du narrateur du Tramway, qui, étrangement, cherche à valider ses souvenirs d'enfance, ou en cherche simplement l'image, le reflet dans le texte proustien. Manifestement, ce narrateur prend La recherche du temps perdu pour une représentation (parfaitement) réaliste, d'où son étonnement lorsqu'il constate une non-conformité entre sa réalité et celle représentée par le texte. En d'autres termes, il prend la fiction de Proust pour la réalité, à l'aune de laquelle devrait donc se conformer la sienne. L'on sait combien Simon a insisté pour dire qu'il n'existait pas d'art réaliste ${ }^{26}$. Le narrateur du Tramway est probablement du

25. Le premier fragment du Tramway comprend un développement sur le «monument aux morts» $(T$, p. 14, 18) qui occupe le centre de la ville, «monumental monument» $(T$, p. 19) devant lequel se réunissent l'après-midi ceux que la mère appelait «avec [...] une sorte de joie mauvaise» (T, p. 19) les «hommes-troncs», leur reprochant sourdement «de s'être sortis, pratiquement coupés en deux mais vivants, de cette guerre qui lui eût arraché le seul homme qu'elle ait jamais aimé» $(T$, p. 20).

26. Simon le déclarait à Madeleine Chapsal en 1967 dans un entretien: «Je ne crois pas au réalisme en littérature pour la bonne raison que c'est impossible: il n'y a pas d'art réaliste, même pour le plus naturaliste des romanciers ou le plus figuratif des peintres. [...] [D] es objets ou des personnages dans un livre, même 
même avis, mais son discours métatextuel, sans le contredire, révèle le phénomène ou le trouble qui l'affecte, et dont rend compte partout ailleurs le roman: le texte de Proust est devenu en partie sa réalité, il a infiltré ses souvenirs personnels. Plus exactement, les images suscitées par le texte proustien se sont confondues dans sa mémoire avec celles préservées de son propre passé, sa propre enfance. De fait, le lecteur familier de l'œuvre de Simon et de celle de Proust aura vite constaté l'exact inverse de ce qu'affirme le narrateur du Tramway, qu'il ne faut donc pas prendre au mot: ce qui frappe, à la lecture du dernier roman de Simon, ce n'est pas la différence avec l'univers proustien, c'est bien plutôt la ressemblance! On pourrait dire que, hormis l'antisémitisme et l'homophobie, ces deux seuls éléments exceptés, donc, tout dans Le tramway rappelle À la recherche du temps perdu.

Anne-Yvonne Julien et Marie Miguet-Ollagnier ont étudié les nombreuses allusions à $L a$ recherche que comprend Le tramway, motifs et procédés puisés dans À l'ombre des jeunes filles en fleurs principalement, et qui apparentent incongrument l'extrême sud de la France où a grandi le narrateur, les plages méditerranéennes où il passait ses vacances, à la station balnéaire normande de Balbec. «Si reliés à des souvenirs personnels que soient [les] paysages [méditerranéens que peint Claude Simon dans Le tramway], ils sont décrits de façon à faire allusion à l'univers de Balbec ${ }^{27}$ ", écrit Marie MiguetOllagnier, notamment par les villas de style normand à colombages qu'on y retrouve et la façon dont les anime le petit groupe d'amis du narrateur, qui «réactive la petite bande de $\mathrm{Balbec}^{28}$ », jeune, énergique et joyeusement insouciante. Ce sont des souvenirs d'enfance contaminés par la lecture du texte proustien et les images qu'il suscite que raconte le narrateur du Tramway. À un point tel qu'il devient impossible de savoir ce qui appartient au texte de Proust (et que le narrateur se serait approprié, aurait indûment fait sien) et ce qui appartient réellement à la mémoire personnelle du narrateur,

décrits minutieusement, ils sont toujours faits de mots et, nous le savons, entre les mots et les choses...» («Claude Simon: "Il n’y a pas d'art réaliste”», La quinzaine littéraire, $\mathrm{n}^{\circ}$ 41, 15-31 décembre 1967, p. 4) Simon affirme de même que «le réalisme ça n'existe pas» dans un entretien ultérieur avec Jo Van Apeldoorn et Charles Grivel ( «De Claude Simon», dans Charles Grivel (dir.), Écriture de la religion. Écriture du roman, Lille, Presses universitaires de Lille, 1979, p. 90).

27. Marie Miguet-Ollagnier, "Claude Simon face à Proust», art. cité, p. 105.

28. Marie Miguet-Ollagnier, "Claude Simon face à Proust», art. cité, p. 105. 
jusqu'au tramway qui donne son titre au roman et qui structure géographiquement et symboliquement son univers.

Sjef Houppermans remarquait déjà en 2003 l'allusion à $L a$ recherche du temps perdu dans le wattman qui ouvre Le tramway, qui en est narrativement et syntaxiquement le premier sujet ${ }^{29}$. Le critique rappelait qu'un wattman joue de même un rôle clé dans Le temps retrouvé, dans la fameuse scène des pavés inégaux, ainsi introduite par Proust: «En roulant les tristes pensées que je disais il y a un instant j'étais entré dans la cour de l'hôtel de Guermantes et dans ma distraction je n'avais pas vu une voiture qui s'avançait; au cri du wattman je n'eus que le temps de me ranger vivement de côté, et je reculai assez pour buter malgré moi contre les pavés assez mal équarris $^{30}$ ", faux mouvement après lequel le narrateur de La recherche ressentira la même félicité que lui avait procurée autrefois, notamment, le goût de la madeleine trempée dans le tilleul ${ }^{31}$. Présidant à la scène des pavés, de la même façon qu'il est «aux commandes d'un processus métaphorique ${ }^{32} »$, chez Simon, personnage «nimbé d'une aura de pouvoir» pour l'enfant ( $T$, p. 12), le wattman «tient le rôle de passeur et $[\ldots]$ se tient sur le seuil d'un autre univers ${ }^{33} »$. Doté de la même ambivalence chez Proust et chez Simon, le wattman, commente Sjef Houppermans, «cach[e] sous ses cris de bonheur un peu

29. La première phrase du Tramway se décline comme suit: «Les graduations en bronze jaune et en relief dessinaient sur le cadran un arc de cercle vers lequel pointait un ergot solidaire de la manette que, pour démarrer ou prendre de la vitesse, le conducteur $[\ldots] »(T$, p. 11$)$.

30. Marcel Proust, Le temps retrouvé [1927], dans À la recherche du temps perdu, éd. Jean-Yves Tadié, Paris, Gallimard, coll. «Bibliothèque de la Pléiade», 1989, t. Iv, p. 445, cité par Sjef Houppermans, «L'homme couché et l'homme debout», dans Jean-Yves Laurichesse (dir.), Claude Simon. Allées et venues, Perpignan, Presses universitaires de Perpignan, 2004, p. 63.

31. Voici la suite de l'extrait du Temps retrouvé (ouvr. cité, p. 445) cité par Sjef Houppermans («L'homme couché et l'homme debout», art. cité, p. 63): «Mais au moment où, me remettant d'aplomb, je posai mon pied sur un pavé qui était un peu moins élevé que le précédent, tout mon découragement s’évanouit devant la même félicité qu’à diverses époques de ma vie m’avaient donnée la vue d'arbres que j'avais cru reconnaître dans une promenade en voiture autour de Balbec, la vue des clochers de Martinville, la saveur d'une madeleine trempée dans une infusion, tant d'autres sensations dont j'ai parlé et que les dernières œuvres de Vinteuil m'avaient paru synthétiser. Comme au moment où je goûtais la madeleine, toute inquiétude sur l'avenir, tout doute intellectuel étaient dissipés [...], se trouvaient levés comme par enchantement.»

32. Didier Alexandre, «Locomotion, transport, émotion dans Le tramway de Claude Simon", Littératures, n 46 (Autour du Tramway de Claude Simon), 2002, p. 82.

33. Sjef Houppermans, «L'homme couché et l'homme debout», art. cité, p. 64. 
trop appuyés que la mort rôde dans ces parages, ce que montreront le Bal de Têtes [chez Proust] et l'évanescence de la mère trop aimée [chez Simon $]^{34} »$. Anne-Yvonne Julien a montré par la suite comment les scènes de vieillesse à l'hôpital du Tramway rejouent celles du «Bal de têtes», le vieil homme avec lequel le narrateur partage une chambre figurant à lui seul l'ensemble des «masques» rencontrés dans le salon du prince de Guermantes. C'est ce qui fait dire à Anne-Yvonne Julien que Le tramway constitue une Recherche $d u$ temps perdu en "modèle réduit ${ }^{35}$ ", reprenant son "principe circulaire ${ }^{36}$ » en vue d'une "ultime circulation en accéléré ${ }^{37}$ » dans l'ensemble de l'œuvre ${ }^{38}$, de la lumière de Combray et Balbec au sombre tragique de l'hôtel de Guermantes.

Plus l'on remonte le fil de l'intertexte, plus le soupçon pèse sur l'authenticité des souvenirs du narrateur du Tramway. Jusqu'à ce qu'on relise le passage d'À l'ombre des jeunes filles en fleurs où le narrateur arrive à Balbec et décrive l'église que, dans son immense déception, il découvre à Balbec-en-terre, à cinq lieues de la plage où il l'avait imaginée, ses pierres «battues par les flots». Plus prosaïquement, relate le narrateur proustien, l'église de Balbec se trouve non loin de la station de chemin de fer, son clocher «se dressa[nt] sur une place où était l'embranchement de deux lignes de tramways, en face d'un Café qui portait, écrit en lettres d'or, le mot "Billard" 39 ". L'enfance du narrateur simonien, comme l'église de Balbec, est elle aussi située à l'embranchement d'une ligne de tramway, caractérisée

34. Sjef Houppermans, «L'homme couché et l'homme debout», art. cité, p. 64.

35. Anne-Yvonne Julien, "L'archive proustienne dans Le tramway de Claude Simon", dans Mireille Calle-Gruber et coll. (dir.), Claude Simon. Les vies de l'archive, Dijon, Éditions universitaires de Dijon, coll. «Écritures», 2014, p. 137.

36. Anne-Yvonne Julien, «L'archive proustienne», art. cité, p. 136.

37. Anne-Yvonne Julien, «L'archive proustienne», art. cité, p. 136.

38. Anne-Yvonne Julien désigne ici l'œuvre simonienne elle-même autant que l'œuvre proustienne, une des hypothèses qu'elle étudie voulant que Le tramway fasse référence aux romans antérieurs de Simon comme autant d'archives (pour un inventaire des références intratextuelles dans Le tramway relevées par Anne-Yvonne Julien, voir "L'archive proustienne», art. cité, principalement p. 135-137).

39. Marcel Proust, À l'ombre des jeunes filles en fleurs, dans À la recherche du temps perdu, éd. Jean-Yves Tadié, Paris, Gallimard, coll. «Bibliothèque de la Pléiade», 1988, t. II, p. 19; je souligne. Simon cite et commente longuement ce passage d'À l'ombre des jeunes filles en fleurs dans une conférence sur Proust qu'il a prononcée en 1980 (voir Claude Simon, "Le poisson-cathédrale», dans Quatre conférences, éd. Patrick Longuet, Paris, Minuit, 2012, p. 9-38, p. 15 pour la citation de l'extrait des Jeunes filles comprenant le mot «tramway»). 
initialement par son «dédoublement» $(T$, p. 16) qui permet d'aller « $[\mathrm{d}]$ ans un sens comme dans l'autre» ( $T$, p. 15). En somme, le wattman et le tramway lui-même pourraient être empruntés à Proust, appartenir au roman et non au vécu, ou être élevés à quelque importance ou rôle symbolique en vertu du texte proustien, au miroir ou à la reconnaissance qu'il aurait offerte aux souvenirs personnels. Le tramway est le roman de cette contamination, du devenir-roman de la mémoire, face opposée mais indissociable du devenir-souvenir du roman.

\section{Le texte romanesque au secours de la mémoire}

Du Jardin des Plantes au Tramway, souvenirs de romans et souvenirs de soi se confondent en images arrachées au temps, temps de la lecture ou temps de la vie. S’ils sont marqués par une même iconicité en vertu de laquelle ils peuvent se substituer les uns aux autres, souvenirs de romans et souvenirs de soi présentent toutefois une différence capitale, peut-être même vitale: les premiers sont arrachés à un texte qui leur survit, qui est préservé dans l'espace matériel du livre. Aussi est-il toujours possible d'aller relire un roman pour en rafraîchir ses souvenirs, en rectifier certains qu'une nouvelle lecture révèle inadéquats au texte, en ajouter d'autres qui les complètent, et ainsi de suite. Les souvenirs personnels ne jouissent pas d'une telle sécurité, d'une telle copie de sauvegarde: le temps vécu, c'est ce que dit Le jardin des Plantes, est irrémédiablement perdu, dès l'instant présent, où il bascule instantanément dans le passé. «Non ce n'est pas [...] l'effet du temps [...] le brouillage de la mémoire»(JP, p. 262), insiste S. face au journaliste, c'est le présent lui-même qui n'est jamais vraiment vécu, sinon comme béance, comme «irréalité » (JP, p. 262).

Aucun dispositif ne peut empêcher cette perte ou remplir cette béance. L'écrivain S. en fait l'expérience lorsqu'il entend sa voix sur le magnétophone utilisé par le journaliste pour enregistrer leur entretien:

Il a dit Voyons si ça marche l'autre jour ce machin n'a pas... Il a appuyé sur une touche, puis une autre et j'ai entendu ma voix en sortir — ou plutôt pas exactement: une voix métallique, timbrée, qui n'est pas la mienne ou du moins celle que j'entends quand je parle $[\ldots]$... Puis la sienne — mais celle-là c'était la même que celle que j'avais entendue ( $J P$, p. 82). 
Cette expérience amène peu après $\mathrm{S}$. à douter de l'«aide-mémoire » (JP, p. 79) que constitue le magnétophone: «Puisque aucune montre ne peut revenir en arrière. Il y avait seulement le magnétophone qui pouvait. Quoique ce ne fut pas exactement ma voix...» (JP, p. 83) Comme l'a montré Didier Alexandre dans une des premières études du Jardin des Plantes, la voix, en rompant la répétition, «inscri[t] une différence ${ }^{40} »$ qui consacre la faillite de l'appareil ou son incapacité à reproduire le passé, «cette saveur d'un instant, faite de sensations et d'émotions ${ }^{41}$ » qui constitue le présent, et qui évolue et se transforme avec celui ou celle qui l'a vécu. À supposer qu'une caméra aurait pu filmer S. dans la débâcle (Le jardin des Plantes se termine d'ailleurs par un scénario préparé par Simon pour une version cinématographique de La route des Flandres qui n'a jamais vu le jour ${ }^{42}$ ), ces images ne lui auraient pas permis d'atteindre son expérience originelle, l'image anachronique de Fabrice del Dongo frappé de stupeur à Waterloo demeurant la plus primordiale, la plus authentique, paradoxalement, de son expérience. Mais S. ne s'arrête pas là pour autant, car un autre recours s'offre à lui: s'il est impossible de «revenir en arrière» $(J P$, p. 83$)$, de remonter le cours du temps, il est possible de remonter le cours $d u$ texte.

Dans un article subséquent sur la tension entre historiographie et autobiographie dans Le jardin des Plantes, Didier Alexandre a étudié la manière dont Simon reconstruit dans ce roman, par le retour sur les événements de mai 1940, «l'instant où tout bascule ${ }^{43}$ », «où

40. Didier Alexandre, «L'enregistrement du Jardin des Plantes», art. cité, p. 11.

41. Didier Alexandre, «L'enregistrement du Jardin des Plantes», art. cité, p. 11.

42. Dans l'édition du Jardin des Plantes comprise dans les Euvres, Alastair Duncan précise (dans Claude Simon, Euvres, ouvr. cité, t. I, p. 1522): «Le scénario de La route des Flandres [qui clôt Le jardin des Plantes] est "imaginaire", selon l'expression même de Simon [...], c'est-à-dire qu'il diffère de celui qu'il a rédigé au début des années 1960. Le scénario donné dans Le jardin des Plantes peut être lu comme une mise en abyme illustrant les principes de construction qui ont déterminé l'ordonnance des morceaux de texte constituant le roman.» Pour une interprétation du rôle du scénario final dans le roman, voir Sophie Charlin, "Que reste-t-il du montage textuel? Efficacité du montage et résidu d'image dans Le jardin des Plantes de Claude Simon", Littérature, $\mathrm{n}^{\circ} 147$ (L'espace du signe), octobre 2007, p. 38-53.

43. Didier Alexandre, "Le renard du jardin. Remarques sur l'insertion du personnage historique dans le récit simonien", dans Jean-Yves Laurichesse (dir.), Le jardin des Plantes de Claude Simon, Perpignan, Presses universitaires de Perpignan, 2000, p. 76. 
s'effectue le passage d'un état ancien à un état nouveau ${ }^{44} »$. Cet instant est situé précisément le 16 mai 1940 entre 17 h et 18 h, alors que les troupes allemandes menées par le général Erwin Rommel opéraient une percée décisive à Clairfayts, après quoi se sont enchaînés à grande vitesse les événements qui ont mené au quasi-anéantissement du régiment de S., puis à la mort de son colonel, retrouvé peu après avec deux survivants sur la route de Solre-le-Château à Avesnes ${ }^{45}$. L'instant charnière de la vie de $\mathrm{S}$. est donc celui qui mène à ce qui sera vécu par lui comme un trauma, comme il le confie au journaliste: «[L]e seul véritable traumatisme qu'il est conscient d'avoir subi $[. .$.$] fut [. .$.$] ce qu'il éprouva pendant l'heure durant laquelle$ il suivit ce colonel [...] sur la route de Solre-le-Château à Avesnes [...] avec la certitude d'être tué dans la seconde qui allait suivre» (JP, p. 223). Dans Le jardin des Plantes, comme l'a défendu dans une lecture récente Jean-François Puff, «Simon essai[e] [...] de comprendre ce qui lui est arrivé. Il s'agit une fois encore, mais de manière nouvelle dans l'œuvre, de tenter par l'écriture de maîtriser un traumatisme enkysté en lui ${ }^{46}$.» Cette manière nouvelle consiste à enchâsser son récit des événements, relatant son expérience personnelle de la débâcle, dans ceux d'autres acteurs ou témoins.

Afin de procéder à cet enchâssement ou ce montage de récits ${ }^{47}$, Simon met à contribution des archives militaires françaises, les

44. Didier Alexandre, «Le renard du jardin», art. cité, p. 76.

45. Ces événements ont été racontés par Simon dans une lettre reproduite sous le titre «Petit historique» dans Anthony Cheal Pugh, «Supplementary History: before, and after La route des Flandres", dans I. Higgins (dir.), The Second World War in Literature, Edinburgh, Scottish Academic Press, 1986, p. 121130; Anthony Cheal Pugh a reproduit à nouveau le «Petit historique» de Simon dans «Claude Simon et la route de la référence», Revue des sciences humaines, $\mathrm{n}^{\circ} 220$ (Claude Simon, dir. Guy Neumann), octobre-décembre 1990, p. 23-45. Voir également l'entretien précédemment cité avec Pierre Bois pour Le Figaro à l'occasion du cinquantenaire de la débâcle de 1940, «La déroute des Flandres», dans Claude Simon, Euvres, ouvr. cité, t. II, p. 1368-1378.

46. Jean-François Puff, "Un dispositif de connaissance. Le jardin des Plantes de Claude Simon et l'historiographie récente de la défaite de 1940 », Cahiers Claude Simon, $n^{\circ} 13$, ouvr. cité, p. 163; l'auteur souligne et précise que le terme "enkysté» est de Simon lui-même.

47. Sur les aspects formels du montage et ses implications esthétiques et éthiques, voir notamment Isabelle Serça, «Le jardin des Plantes: une "composition en damier" ", Littératures, n 40, ouvr. cité, p. 59-77; Nathalie Piégay, "Mélancolie du montage. Le jardin des Plantes de Claude Simon», dans Emmanuel Bouju (dir.), L'engagement littéraire, Rennes, Presses universitaires de Rennes, 2005, p. 283-291; Mireille Calle-Gruber, Le grand temps. Essai sur l'œuvre de Claude 
Carnets du général Rommel et les Mémoires de guerre de Churchill, qu'il cite ou paraphrase dans des fragments dont la juxtaposition traduit la simultanéité des événements. Dans la deuxième partie du Jardin des Plantes, c'est cet enchâssement qui permet de reconstituer «l'instant où tout bascule ${ }^{48}$ », de lui conférer une « intelligibilité historique », comme l'a montré Didier Alexandre, par la conjonction «de trois niveaux de temporalités - Rommel, S., Churchill ${ }^{49} »$ : Rommel à Clairfayts prenant de force la France $(J P$, p. 188), S. à Joncret constatant l'arrêt de sa montre brisée par une explosion (JP, p. 167) et Churchill au Quai d'Orsay se faisant dire par le général Gamelin que la France ne dispose plus d' "aucune masse de manœuvre» (JP, p. 181). Tout cela survient simultanément, autour de cinq heures de l'après-midi, le 16 mai 1940, c'est-à-dire le début de la fin, pour S., les aiguilles de sa montre "arrêtées à $17 \mathrm{~h} 45$ » (JP, p. 167). Ce qu'on n'a pas encore vu, me semble-t-il, c'est le rôle clé joué dans cet enchâssement par les dix-huit fragments de Sodome et Gomorrhe insérés aussi dans la deuxième partie du Jardin des Plantes, insertion qu'on a souvent commentée mais jamais sous cet angle. Aux trois trajectoires ou temporalités identifiées par Didier Alexandre, il faudrait en effet en ajouter une quatrième: celle de Proust (ou Marcel). Elle est bien évidemment hétérogène, dans l'ensemble, discordante, mais non moins importante: la compréhension ou l'intelligibilité à laquelle tend Le jardin des Plantes, la maîtrise du traumatisme procèdent de la conjugaison des récits de Rommel, de Churchill et de S., et, proposerais-je, du texte de Proust.

Le texte de Proust fait bel et bien partie de l'enchâssement, la séquence initiale de la deuxième partie du Jardin des Plantes présentant dans cette suite exacte " [1] es trajectoires respectivement suivies par S. et R.» (JP, p. 159), le «Premier ministre» (JP, p. 160) anglais à l'«éternel cigare» et l' «éternel [visage de] gros bébé» $(J P$, p. 161), puis Proust, Simon évoquant un extrait d'À la recherche du temps perdu qui se passe très précisément, il le signale d'emblée, en mai vers cinq heures de l'après-midi (de la même façon que l'entretien avec le journaliste, à l'autre bout du siècle, se passe aussi en mai entre $17 \mathrm{~h}$ et $18 \mathrm{~h})$ :

Simon, Villeneuve-d'Ascq, Presses universitaires du Septentrion, 2004, p. 42-45 et p. 145-190.

48. Didier Alexandre, «Le renard du jardin », art. cité, p. 76.

49. Didier Alexandre, «Le renard du jardin », art. cité, p. 76. 
Quoique Proust ne donne pas d'indications précises, on peut déduire du contexte qu'il doit être environ cinq heures de l'aprèsmidi lorsque accompagnée de sa bru, née Legrandin, la marquise de Cambremer [...] vient s'installer avec le narrateur et Albertine sur la terrasse du Grand-Hôtel. "Nous regardions la mer calme où des mouettes éparses flottaient comme des corolles blanches", écrit Proust en effet, ce qui donne à penser (comme on pourra le voir au cours du récit où les mouettes changent peu à peu de couleur tandis que l'heure avance) qu'au moment où s'installent la marquise et ses amis la lumière (on est au mois de mai) ne se teinte pas encore de ces irisations qu'elle prend au fur et à mesure que le soleil décline (JP, p. 161-162; je souligne).

Dans la suite de l'extrait cité par Simon, le narrateur de La recherche entame la conversation avec les dames de Cambremer en évoquant la beauté et la blancheur des mouettes, qu'il compare à des «"nymphéas"» (JP, p. 161), image qui introduit une discussion sur l'art où sont comparés Monet et Poussin, la jeune madame de Cambremer qualifiant ce dernier de " Le véritable objet de cette conversation, commente Simon dans Le jardin des Plantes, est "l'écoulement du temps», que Proust "ren[d] sensible à travers les successives informations qu'il donnera sur les couleurs changeantes des mouettes à mesure que le soleil décline[,] [...] pendant que se poursuivra l'échange des réflexions sur l'art plus ou moins influencées par la mode et par l'âge (ce qui est encore une façon d'évoquer le temps)» (JP, p. 175). L'extrait cité de Sodome et Gomorrhe porte sur le temps qui passe, effectivement, mais aussi sur la possibilité d'en remonter le cours en juxtaposant des images ou des tableaux (sur le modèle de la bande cinématographique), ou encore, peut-être, des fragments de texte, de la même manière que sont disposés les fragments de Sodome et Gomorrhe dans Le jardin des Plantes.

Ce n'est pas par simple assonance que Simon insère la conversation mondaine de Sodome et Gomorrhe dans sa reconstitution de la débâcle, de l'instant où, pour lui, tout a basculé, où le temps s'est arrêté. Plus fondamentalement, le texte de Proust tient lieu de la mémoire défaillante, trouée en son fondement, de S. L'explication de cet étrange phénomène est dans Le tramway, qui en donne la clé: puisque ses souvenirs de La recherche sont devenus en partie, indistinctement, ses souvenirs personnels, puisque les deux mémoires, 
celle de La recherche et celle de son propre passé, se sont contaminées, le texte proustien est devenu en partie le gardien de la mémoire personnelle du narrateur simonien. Aussi, à défaut de pouvoir revenir en arrière, se butant sur le "brouillard d'irréalité» de l'événement traumatique, ou sur la béance originelle que recouvre le souvenir de Fabrice del Dongo, le narrateur simonien va remonter le cours du texte proustien - texte que Simon n'a jamais cité aussi fidèlement par ailleurs, c'est dire que c'est vraiment le texte proustien qui est présent, ici, déployé dans plusieurs fragments successifs. La contamination de la mémoire personnelle par le souvenir du roman, si elle fragilise l'individu, l'intégrité de sa mémoire, est ici, tel qu'annoncé en introduction, une chance: cherchant avec le journaliste comment il s'est senti à l'instant où tout a basculé en mai 1940, S. trouve la réponse en partie dans le texte proustien, dans un extrait qui se passe précisément en mai à la même heure et sous le même soleil, dans le même temps (calendaire, météorologique). C'est, cette fois, le texte romanesque qui tient lieu de la mémoire personnelle. Le texte de Proust, en ce sens, fait bel et bien partie de la reconstitution historique que tente Le jardin des Plantes: il accompagne S. comme une copie de secours de sa mémoire, copie altérée certes, plus encore que la voix enregistrée sur la bande du magnétophone, mais offrant peut-être par là la chance d'une relance.

Je poserai comme dernière hypothèse que c'est de cet assemblage comprenant le texte de Proust que sort le mot «mélancolie», si difficilement prononcé par S. et qui est le fin mot de son expérience en cette heure traumatique de son existence: «[E]t à la fin il dit Mélancolie [...] quelque chose de violent qui protestait, furieux, bâillonné mais hurlant: Jamais je n'avais tant désiré vivre, jamais je n'avais regardé avec autant d'avidité, d'émerveillement, le ciel, les nuages, les prés, les haies...» (JP, p. 299-303) Didier Alexandre définit cette mélancolie comme la "perception d'une rumeur», bruit de «la force destructrice de l'homme, réalisée dans Rommel, qui accomplit la ruse de la raison hégélienne, au point de n'être plus que négativité pure, boulevers[ant] le monde du dehors, [...] bruit anodin, produit par des éléments qui se neutralisent ${ }^{50} »$. La force négatrice, destructrice de Rommel, "agent du désastre ${ }^{51}$ », a cependant ceci de positif pour S., relève Didier Alexandre, qu' « elle enseigne que

50. Didier Alexandre, «Le renard du jardin », art. cité, p. 82.

51. Didier Alexandre, «Le renard du jardin», art. cité, p. 85. 
l'homme est mortel, dans tous les sens du terme, puisqu'il donne la mort et la reçoit ${ }^{52}$ », qu'il recèle «la violence contenue dans la matière - une matière qui englobe l'homme et le monde ${ }^{53}$ ». La figure de Rommel serait dès lors associée à une «éthique de la force» face à laquelle Churchill serait mis à distance: «Churchill incarne le passé, d'un point de vue éthique et esthétique: après la guerre, il fera une peinture académique, inspirée des impressionnistes[, comme le mentionne Le jardin des Plantes (JP, p. 169)]. Rommel, par son éthique de la force, incarne la rupture et ouvre sur le futur ${ }^{54} »$.

Fondamentalement issue de cette expérience de la guerre, de la rumeur qui se fait toujours entendre et qui est «indissociable de la mort ${ }^{55}$ ", l'écriture de Simon pratiquera elle aussi la rupture, luttant contre les modèles académiques, à l'opposé de Churchill dans sa peinture. Mais elle le fera, et c'est là que le texte proustien tient une place primordiale dans l'ensemble à quatre voix ou plus précisément à quatre textes qu'orchestre Le jardin des Plantes, à la manière de Proust: en différant la fin, en reportant toujours la rupture, la séparation définitive. Le texte proustien serait dès lors convoqué dans la deuxième partie du Jardin des Plantes pour son extraordinaire puissance de retardement, pour sa «dynamique ralentissante [...] [qui] serait, autant qu'une manière de représenter la vie, un déni de la nécessité de mourir ${ }^{56} »$. Pour réécrire ou reconstituer son avancée funeste vers les armées de Rommel, S. se pare du texte de Proust, s'en fait une sorte de bouclier: Proust est l'allié qu'il choisit, contre Churchill qui est mis à distance, pour sortir vivant de la bataille. Face aux tirs des armées de Rommel, à la «froide méthode d'anéantissement ${ }^{57}$ " du général, Simon oppose la capacité proustienne à tirer les choses les plus infimes de l'oubli, ce que disait déjà le passage du Temps retrouvé mis en exergue de La bataille de Pharsale en 1969:

[J]e fixais avec attention devant mon esprit quelque image qui m'avait forcé à la regarder, un nuage, un triangle, un clocher, une fleur, un caillou, en sentant qu'il y avait peut-être sous ces signes quelque chose de tout autre que je devais tâcher de découvrir, une

52. Didier Alexandre, «Le renard du jardin», art. cité, p. 82.

53. Didier Alexandre, «Le renard du jardin», art. cité, p. 80.

54. Didier Alexandre, «Le renard du jardin», art. cité, p. 81.

55. Didier Alexandre, «Le renard du jardin», art. cité, p. 82.

56. Christophe Pradeau, «Le roman a le temps», art. cité, p. 87.

57. Jean-François Puff, «Un dispositif de connaissance», art. cité, p. 173. 
pensée qu'ils traduisaient à la façon de ces caractères hiéroglyphes qu'on croirait représenter seulement des objets matériels ${ }^{58}$.

L'attention dont témoigne le texte proustien est mise au service de la survie du personnage simonien dans Le jardin des Plantes.

À partir du moment où roman et vie sont devenus indistincts, ont fusionné dans une étrange osmose, retarder la fin de l'un, c'est repousser la fin de l'autre: tant que dure le texte romanesque, dure la vie, ou à tout le moins est repoussé le moment de la mort. Le texte romanesque, en cela, et le texte proustien en particulier, est bien le contraire de l'image, dont Didier Alexandre rappelle, citant Blanchot, qu'elle est "la chose comme éloignement" 59 ", ou, selon les mots de Bérénice Bonhomme, «séparation d'avec les choses par l'intermédiaire de l'écran du temps ${ }^{60}{ }$. L'image est séparation, arrachement au temps (celui de la vie, celui du roman). Le déploiement de l'image par et dans le texte est une manière de retarder l'ultime

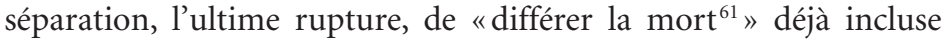
dans le souvenir, dans l'instantané devenir-image du passé.

Un des deux exergues du Tramway est emprunté à Proust, tiré des pages célèbres de "Combray» où le narrateur raconte ses souvenirs de lecture d'enfance: «[L]'image étant le seul élément essentiel, la simplification qui consisterait à supprimer purement et simplement les personnages réels serait un perfectionnement décisif.» ( $T$, exergue, p. 9) L'extrait ne le laisse pas voir, mais le passage cité porte en fait sur le travail du romancier et son rapport aux personnages réels, opposés aux personnages imaginaires ou inventés:

[T] ous les sentiments que nous font éprouver la joie ou l'infortune d'un personnage réel [c'est-à-dire d'une personne réelle,

58. Marcel Proust, Le temps retrouvé, ouvr. cité, p. 457; cité par Claude Simon, La bataille de Pharsale, Paris, Minuit, 1969, p. 99.

59. Didier Alexandre, "Le renard du jardin », art. cité, p. 84; Didier Alexandre cite Maurice Blanchot dans L'espace littéraire [1955], Paris, Gallimard, coll. «Idées », 1982, p. 347.

60. Bérénice Bonhomme, "Claude Simon: une contestation du texte par l'image», Cahiers de narratologie [En ligne], $\mathrm{n}^{\circ} 16$ (Les limites du récit, dir. Jean-Paul Aubert), 2009, mis en ligne le 25 mai 2009, consulté le 12 novembre 2019, URL: https://journals.openedition.org/narratologie/1025.

61. Didier Alexandre, «Le renard du jardin », art. cité, p. 84. 
dans la réalité] ne se produisent en nous que par l'intermédiaire d'une image de cette joie ou de cette infortune; l'ingéniosité du premier romancier consista à comprendre que dans l'appareil de nos émotions, l'image étant le seul élément essentiel, la simplification qui consisterait à supprimer purement et simplement les personnages réels serait un perfectionnement décisif ${ }^{62}$.

Le conditionnel me semble implacable, ici: le romancier a compris que la suppression des personnages réels serait un perfectionnement. La réalité, c'est que cette suppression n'est pas possible, pas tout à fait: les personnages de romans, chez Proust, ne sont jamais très loin d'une ou de plusieurs personnes réelles dont ils synthétisent les traits. On retrouve le même conditionnel dans un autre passage du Tramway lié à Proust, que j'ai cité auparavant, et qui me semble se rattacher à l'exergue: "On s'explique mal pourquoi, torturé par la jalousie, le narrateur (qui, comme Proust le laisse entendre, pourrait s'appeler Marcel) $[\ldots] »(T$, p. 58 ; je souligne). Le narrateur de $L a$ recherche pourrait s'appeler Marcel, c'est-à-dire que le personnage qu'il constitue, s'il n'équivaut pas à Marcel Proust, n'est pas non plus complètement séparé de lui, n'est pas si différent. Il en va bien sûr de même du narrateur simonien, dans les romans «à base de vécu ${ }^{63}$ » de Simon. Ainsi, le romancier (Proust) a compris quelque chose (c'està-dire que la suppression des personnages réels serait un perfectionnement décisif), mais il fait autre chose: il ne supprime pas, pas tout à fait, les personnages réels. En d'autres termes, il ne s'abandonne pas à l'image, ce qui reviendrait à consacrer la séparation avec les "êtres réels ${ }^{64}$ », ceux dont le roman, justement, prolonge indéfiniment le deuil, «poids mort que notre sensibilité ne peut soulever ${ }^{65}$ ».

Si la même chose peut être dite du Jardin des Plantes, qui convoque la puissance de retardement du texte proustien et redéploie dans un roman-somme, comme pour ne jamais en finir, l'histoire de 1940 racontée maintes et maintes fois, il n'en va pas de même du Tramway. Le romancier, dans ce dernier opus simonien,

62. Marcel Proust, Du côté de chez Swann, dans À la recherche du temps perdu, éd. Jean-Yves Tadié, Paris, Gallimard, coll. «Bibliothèque de la Pléiade», 1987, t. I, p. 84 ; je souligne.

63. L'expression est de Simon, qui affirme: «Plutôt qu'autobiographiques, je préfere dire que mes livres sont à base de vécu.» («"Et à quoi bon inventer?”, entretien avec Marianne Alphant, Libération, 31 août 1989, p. 24)

64. Marcel Proust, Du côté de chez Swann, ouvr. cité, p. 84.

65. Marcel Proust, Du côté de chez Swann, ouvr. cité, p. 84. 
à l'opposé de ce qu'il a fait toute sa vie durant, effectuerait (ou presque) le perfectionnement romanesque décisif: il s'abandonnerait à l'image, c'est-à-dire qu'il renoncerait à la puissance de retardement du roman, qu'emblématise le texte proustien. Et, de fait, si Le tramway expose une mémoire personnelle contaminée par le texte proustien, il est dans sa forme le roman le moins proustien produit par Simon, opérant par là une séparation. En permettant, comme le dit Anne-Yvonne Julien, une «circulation en accéléré» dans À la recherche $d u$ temps perdu, dont il reprend le principe circulaire pour le mettre à nu, en quelque sorte, Le tramway laisse de côté «le long milieu» du roman proustien, que Proust a voulu allonger de l'intérieur jusqu'à la fin de sa vie, jusqu'à ce que la mort mette un terme à son entreprise. En ce sens, Le tramway manifeste bien «un refus du déni de la mort ${ }^{66}$ ", auquel il oppose «une image de l'affirmation de la mort $^{67}$ ", comme l'écrit Pascal Mougin dans un article sur le deuil de la mère dans le dernier roman de Simon. Dans Le tramway, comme l'écrit Pascal Mougin, «le deuil est fait ${ }^{68} »:$ «[La] douleur de l'acceptation de la perte [...] ne "travaille" plus le narrateur et ne déborde plus dans le récit ${ }^{69} »$. Accomplissant le deuil de la mère, Le tramway accomplirait aussi celui du roman, du roman proustien en particulier, abandon qui est aussi installation dans l'image, faceà-face ultime mais apaisé avec le devenir-image de toute chose, où s'apprécie encore l'empreinte de ce qui a été.

66. Pascal Mougin, «La mère, la mère toujours recommencée», dans Jean-Yves Laurichesse (dir.), Claude Simon. Allées et venues, ouvr. cité, p. 193.

67. Pascal Mougin, «La mère, la mère toujours recommencée», art. cité, p. 192; je souligne.

68. Pascal Mougin, «La mère, la mère toujours recommencée», art. cité, p. 194.

69. Pascal Mougin, «La mère, la mère toujours recommencée», art. cité, p. 194. 